\title{
Drug-Induced Liver Injury: Clinical Evidence of N-Acetyl Cysteine Protective Effects
}

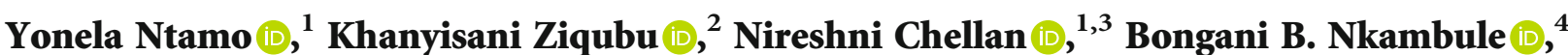 \\ Tawanda M. Nyambuya $\mathbb{D}^{5},{ }^{5}$ Sithandiwe E. Mazibuko-Mbeje $\mathbb{D}^{D},{ }^{2}$ Kwazikwakhe B. Gabuza ${ }^{(D)}{ }^{1}$ \\ Fabio Marcheggiani $\mathbb{D}^{6}{ }^{6}$ Luca Tiano $\mathbb{D}^{6},{ }^{6}$ and Phiwayinkosi V. Dludla $\mathbb{D}^{1}$ \\ ${ }^{1}$ Biomedical Research and Innovation Platform, South African Medical Research Council, Tygerberg 7505, South Africa \\ ${ }^{2}$ Department of Biochemistry, North-West University, Mmabatho 2745, South Africa \\ ${ }^{3}$ Division of Medical Physiology, Faculty of Medicine and Health Sciences, Stellenbosch University, Tygerberg 7505, South Africa \\ ${ }^{4}$ School of Laboratory Medicine and Medical Sciences, University of KwaZulu-Natal, Durban 4000, South Africa \\ ${ }^{5}$ Department of Health Sciences, Namibia University of Science and Technology, Windhoek 9000, Namibia \\ ${ }^{6}$ Department of Life and Environmental Sciences, Polytechnic University of Marche, Ancona 60131, Italy
}

Correspondence should be addressed to Phiwayinkosi V. Dludla; pdludla@mrc.ac.za

Received 12 August 2021; Revised 12 November 2021; Accepted 22 November 2021; Published 6 December 2021

Academic Editor: Daniela Giustarini

Copyright ( 2021 Yonela Ntamo et al. This is an open access article distributed under the Creative Commons Attribution License, which permits unrestricted use, distribution, and reproduction in any medium, provided the original work is properly cited.

Oxidative stress is a key pathological feature implicated in both acute and chronic liver diseases, including drug-induced liver injury (DILI). The latter describes hepatic injury arising as a direct toxic effect of administered drugs or their metabolites. Although still underreported, DILI remains a significant cause of liver failure, especially in developed nations. Currently, it is understood that mitochondrial-generated oxidative stress and abnormalities in phase I/II metabolism, leading to glutathione (GSH) suppression, drive the onset of DILI. N-Acetyl cysteine (NAC) has attracted a lot of interest as a therapeutic agent against DILI because of its strong antioxidant properties, especially in relation to enhancing endogenous GSH content to counteract oxidative stress. Thus, in addition to updating information on the pathophysiological mechanisms implicated in oxidative-induced hepatic injury, the current review critically discusses clinical evidence on the protective effects of NAC against DILI, including the reduction of patient mortality. Besides injury caused by paracetamol, NAC can also improve liver function in relation to other forms of liver injury such as those induced by excessive alcohol intake. The implicated therapeutic mechanisms of NAC extend from enhancing hepatic GSH levels to reducing biomarkers of paracetamol toxicity such as keratin-18 and circulating caspase-cleaved cytokeratin-18. However, there is still lack of evidence confirming the benefits of using NAC in combination with other therapies in patients with DILI.

\section{Introduction}

Undoubtedly, the increased use of drugs in medical care has become the prominent cause of liver injury [1,2]. Thus, being the largest organ within the body, the liver remains crucial for neutralizing detrimental substances that may enter the human system leading to tissue damage. Notably, liver cells play an active role in absorbing and eliminating potentially damaging constituents, including bacterial products or drugs transported by the portal blood or microorganisms [3]. In fact, evaluation of liver chemistry tests has become a common and routine procedure for clinicians to diagnose liver disease or injury, including monitoring adverse effects that may be induced by drug reactions [4]. Certainly, drug-induced liver injury (DILI, commonly known as drug-induced hepatotoxicity) has been the main determining factor for drug restriction or withdrawal from the pharmaceutical market [5]. Accordingly, different international working groups such as the Council for International Organizations of Medical Sciences have provided essential tools to detect, diagnose, and manage an array of liver diseases, including drug-induced liver injury (DILI) [6].

DILI broadly describes any injury to the liver that might occur as a result of prescribed medication or even dietary 
supplements that may develop from asymptomatic liver test elevations to induce acute liver failure [7]. The latter is by far the most common manifestation, said to be responsible for more than $90 \%$ cases of DILI $[8,9]$. This explains progressive research being undertaken to develop novel biomarkers that can predict or diagnose DILI [10]. For example, circulating reactive intermediary metabolites are discussed as potential biomarkers to identify the initial pathological events involved in drug-induced hepatoxicity $[9,11]$. Nevertheless, it was long established that $\mathrm{N}$-acetyl-p-benzo-quinone imine (NAPQI), a reactive metabolite of paracetamol (also systemically referred to as acetaminophen), may drive oxidative stress-induced hepatoxicity by depleting intracellular glutathione (GSH) levels [12]. As the liver remains fundamental for detoxification, it is more susceptible to oxidative stress-induced damage, especially as a result of suppressed intracellular antioxidant defence systems [13].

Indeed, oxidative stress plays a key role in the pathogenesis of DILI $[11,14,15]$, thus indicating that alternative therapies with abundant antioxidant properties are a feasible strategy to counteract this devastating outcome and protect against liver injury [13]. Because of its strong antioxidant properties and its known capacity to enhance intracellular GSH levels, N-acetyl cysteine (NAC) has attracted a lot of interest as a therapeutic agent against diverse diseases [16-19], besides being a drug of choice to protect against paracetamol-induced liver injury [19]. In addition to enhancing GSH levels, recent developments suggest NAC can modulate other mechanisms of oxidative stress, including reducing endoplasmic reticulum stress (ER) or improving mitochondrial function, to protect against liver injury $[20,21]$. Thus, the current review explores mechanisms of oxidative stress implicated in the development of DILI. Importantly, a systematic search, through major search engines such as PubMed and Google Scholar, was conducted to identify evidence from randomized clinical trials (RCTs) reporting on the impact of NAC infusion in patients with DILI. Please note that the methodology and motivation for the study selection were based on modifying already published protocols that aim to understand and update the therapeutic effects of NAC against diverse disease complications $[22,23]$. Moreover, information relevant to the therapeutic potential of NAC to modulate oxidative stress to protect against DILI, beyond paracetamol-induced liver injury, is also discussed.

\section{An Overview on Drug-Induced Liver Injury}

Great strides in industrialization and drug development have come with benefits as well as inevitable risks. Some of the risks include the development of drug compounds that have a toxic effect on humans, and animals, post-market. A major contributing factor that continues to torment the pharmaceutical industry is the clinical safety issues post-market which are often associated with adverse side effects related to liver injury and causes costly drug withdrawals [24, 25]. In this regard, DILI has been a major topic in the fields of hepatology and toxicity. This condition is a rare lifethreatening disease that can be described as hepatotoxicity resulting from the use various pharmaceutical agents, or herbs, subsequently prompting liver dysfunction, successive chronic liver failure, and/or acute liver injury in the absence of other etiologies [9, 26-29]. DILI is a common cause of both acute liver injury and chronic liver failure, presenting itself as a public health concern. In general, DILI represents a broad spectrum of clinical manifestations such as abnormal elevation of liver enzymes, hepatitis, hepatocellular necrosis, cholestasis, fatty liver, and liver cirrhosis $[9,30$, 31]. However, the clinical symptoms of DILI are quite hard to distinguish from other hepatic disorders, and unfortunately no age is exempt, albeit the risk is higher in adults and the elderly. Because of its broad-spectrum manifestations, a specific criterion is used to identify DILI using blood samples from patients, in general the criterion includes the following: a 3-5 times increase in liver enzymes such as alanine aminotransferase (ALT), aspartate aminotransferase (AST), alkaline phosphatase (ALP), or bilirubin, above their upper limit of normal [30, 32-35]. Recent studies show that various over-the-counter drugs induce DILI. For example, acetaminophen is considered the most common cause of DILI in the Western world [36, 37], while other medications such as ibuprofen [38] and antituberculosis drugs [30, 39, 40] may drive complications related to the development of DILI. Moreover, an important clinical problem has been reported due to the surge in immunotherapy-related hepatotoxicity caused by anticancer drugs and autoimmune suppressive drug therapies [41-43]. This of course is expected as immune-related side effects for enhancing the body's immune response to malignancies, which involves unwanted inflammation. Moreover, although it is widely accepted that approximately $80 \%$ of the Asian and African populations use traditional medicine [44], which can also possibly contribute to the development DILI [45], very few population-based studies are available to provide reliable statistics.

Perhaps the major setback with DILI is the limited information regarding its incidences in population studies. Despite that, a few population-based studies have been conducted and reported around the world. For example, a study by Björnsson and coworkers has reported that in Iceland, between 2010 and 2011, crude annual DILI incidence rate was 19.1 cases per 100,000 inhabitants, with $75 \%$ cases caused by a single prescription medication [32]. While in the United States, DILI has been known to account $50 \%$ of all cases of acute liver failures and nearly $10 \%$ of total cases of acute hepatitis, carrying a mortality rate of approximately $10 \%$ [46-48]. A study by Shen and coworkers reported that the annual incidence in the general population was estimated to be 23.80 per 100,000 persons in a study conducted in mainland China [31]. In France, the estimated DILI incidences are approximately 13.9 per 100,000 persons according to the French population-based study conducted [49]. In a study from India conducted in a single center, it was reported that DILI contributed to $2.5 \%$ of hepatobiliary admissions with a gradual increase in the numbers over the years [40].

2.1. Pathogenesis of Drug-Induced Liver Injury. The pathogenesis of DILI typically implicates the involvement of a toxic drug/metabolite that either directly affects the biochemistry of 
the cell or elicits an immune response. In any case, the resultant cell death is the event that leads to the clinical manifestation of DILI symptoms. The exact cellular and molecular mechanisms involved in the pathogenesis of DILI are currently poorly understood; however, several studies have divided the mechanisms into direct toxicity which is from drugs or their metabolites directly damaging the cytoplasm membrane, ER, or other organelles of the liver cells. While some studies propose an indirect toxicity injury where toxins first interfere with the metabolic pathways or metabolism of macromolecules, such as proteins or DNA, thus provoking generation of oxidative stress, hinder mitochondrial function that eventually changes the cell structure and eventually causes cell death $[9,26-28,50,51]$. Due to the diverse nature of the pathology, especially when it involves unintentional overdosing, it becomes difficult to manage or treat complications linked with DILI. The most practical solution would perhaps be to exercise caution during the empirical treatment of certain diseases such as tuberculosis, given the implications of antituberculosis drugs in the high incidence of severe DILI.

2.2. Translational Gaps of Experimental Studies. Clearly, early research has indicated that different animal species, including guinea pigs, hamsters, and rats, were used to study the pathogenesis of DILI (reviewed evidence [52]). Extending beyond these in vivo systems, primary mouse hepatocytes, metabolically competent cell lines, and mouse models have become increasingly applicable to understand the pathological mechanisms implicated in the development of DILI [52-54]. Notably, primary hepatocytes and their adherent cultures represent an easy-to-handle in vitro system to study the complications of DILI; however, arising challenges consistently implicate the process of dedifferentiation during primary hepatocyte isolations [55]. The latter describes an undesirable phenomena that negatively affects hepatocyte morphology and functionality, usually in response to the activation of proliferative and inflammatory mechanisms during the two-step collagenase perfusion method [55]. In fact, this process may interfere with the understanding of vital molecular mechanisms, as dedifferentiation is linked with the suppression of relevant liverspecific genes [56]. Thus, while primary hepatocyte cultures may be useful to explore classical features of DILI, including those implicating oxidative stress-induced liver injury [55, 56], some limitations persist regarding their reliability and routine use. Interestingly, recent laboratory technology innovations or advances such as those making use of three-dimensional (3D) experimental models have become important. Such innovative experimental models have become relevant to predict potential cytotoxicity during the early stages of drug development. For example, through their capacity to mimic the in vivo microenvironment, 3D experimental models have become a useful tool to study pharmacodynamics, which is essential for the early detection of possible toxic effects of potential drugs before reaching human testing [57-62]. Beyond understanding the dynamics of drug-induced toxicology, 3D experimental models have also become important to decipher pathophysiological mechanisms implicated in the development of DILI, espe- cially the role of oxidative stress as it remains one of major therapeutic targets to reverse complications linked with DILI [57-64]. However, despite the progress that has been made with the 3D cocultures, preclinical (animal) models, especially the application of mice remain the common experimental system that is currently used to study the pathogenesis of DILI [52-54]. Notably, rodents seem to easily develop complications such as mitochondrial damage or oxidative stress-linked hepatocellular apoptosis in response to drug exposure, and this has been easily correlated to mechanisms identified in humans [52-54]. Moreover, animal models remain relevant for understanding the modulation of immune system, another important feature in the development of DILI which cannot be easily explored through in vitro cultured cells [52-54]. Nonetheless, it appears that additional research is still required to better understand certain experimental models, such as primary mouse hepatocytes, the 3D-cocultures, and in vivo rodent models, to enhance their applicability and easy translation to mechanisms identified in patients.

\section{Oxidative Stress in Drug-Induced Liver Injury}

Oxidative stress is well-recognized as a predominant pathogenic process for the development of acute liver failure. As a result, there is already increased evidence describing the implications of oxidative stress in the pathophysiology of diverse liver conditions $[65,66]$, which poses a higher risk of developing acute liver failure upon exposure to hepatotoxic drugs [67]. Different drug compounds are thought to have different modes in DILI pathogenesis, including prompting mitochondrial dysfunction, as part of the characteristic feature of oxidative stress driven toxicity $[34,68]$. Thus, oxidative stress represents an attractive therapeutic target to protect the liver against DILI. As a result, growing body of knowledge has reported on the diverse pathophysiological mechanisms implicating oxidative stress and suppressed cellular antioxidant defence systems in the development of DILI.

Generally, oxidative stress is a well-studied pathological feature caused by an imbalance between the generation of highly reactive molecules and their detoxification by the intracellular antioxidant defence systems. Under disease conditions, the immoderate production of radical molecules such as reactive oxygen species (ROS) usually cause direct damage to the biomolecules and eventually impair biochemical processes and cause cellular injury [69]. Although the liver is well-equipped with substantial antioxidant defences such as GSH, glutathione peroxidase (GPx), superoxide dismutase (SOD), and catalase to scavenge ROS, the metabolism of certain drugs can deplete the defence antioxidants and induce a state of oxidative stress that precede adverse liver injuries [66]. As a prime example, acetaminophen, which is a widely used pharmaceutical drug to moderate pain, is well-studied in the context of DILI, as it remains the leading cause of drug-induced liver failure in many countries, when used above the recommended dose (Figure 1) [70]. Normally, at an optimal dose, acetaminophen is effectively metabolized by CYP2E1 and CYP1A2 


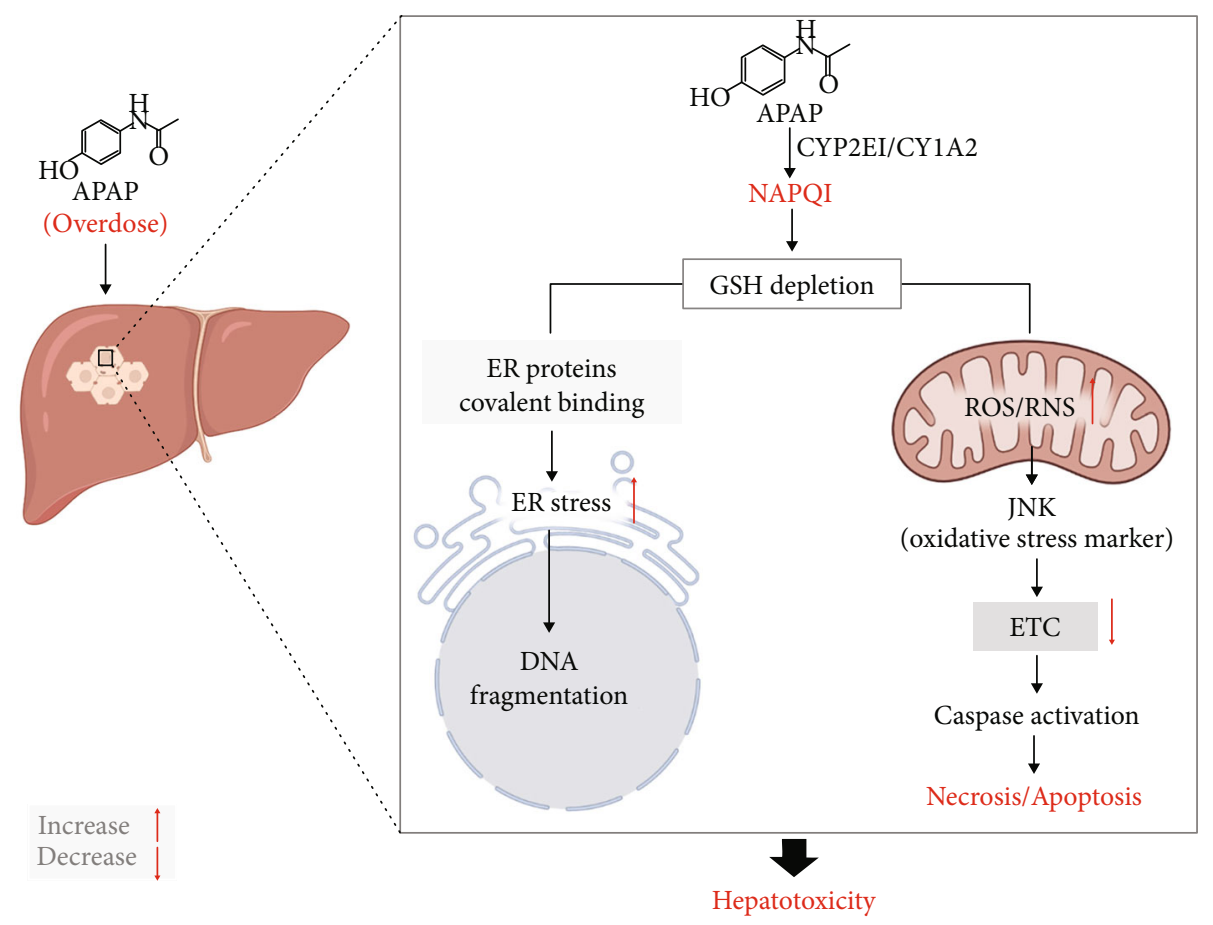

FIGURE 1: The mechanisms showing the implication of oxidative stress in the pathophysiology of paracetamol (acetaminophen) liver injury. Briefly, as a consequence of paracetamol overdose, remaining nontherapeutic doses of paracetamol become metabolized by membranebound enzymes such as CYP2E1 and CYP1A2 to its reactive intermediate toxic metabolite, NAPQI. The generated NAPQI forms mitochondrial protein adducts which in turn are implicated to play a major role in driving oxidative stress-induced hepatoxicity through rapid conjugation with GSH and subsequently initiating signaling cascades resulting in programmed cell death. Abbreviations: CYP2E1: cytochrome P450 2E1; CYP1A2: cytochrome P450 1A2; NAPQI: N-acetyl-p-benzo-quinone imine; GSH: reduced glutathione; ROS: reactive oxygen species; RNS: reactive nitrogen species; JNK: c-Jun N-terminal kinase; ETC: electron transport chain.

enzymes to form a toxic metabolite, NAPQI, which is known to play a major role in drive oxidative stress-induced hepatoxicity through rapid conjugation with GSH [71]. Thus, as the reaction may be accelerated during periods overdose, elevated levels of NAPQI may deplete mitochondrial GSH content, resulting in the defective removal of ROS and increased generation of oxidative stress [71]. Briefly, in a well described mechanism, paracetamol is broken down by membrane-bound cytochrome p450 enzymes such as CYP2E1, CYP1A2 and CYP3A4 to its reactive intermediate, NAPQI which is known to covalently bind to mitochondria and cause direct hepatic toxicity by prompting increased generation of ROS and reactive nitrogen species (RNS) that ultimately drive apoptosis and necrotic cell death $[1,72$, 73]. Furthermore, in rather diverse molecular mechanisms that drives the pathogenesis of DILI, oxidative stress may be induced by other stimuli that lead to hepatic cellular death. For example, excessive alcohol consumption can also induce increased production of ROS, which reacts with essential cellular biomolecules such as lipids, proteins, or nucleotides, that ultimately cause depletion of intracellular antioxidants, further driving the detrimental effects of oxidative stress-related hepatic damage [74, 75]. In agreement, Jin et al. [76] recently demonstrated that administration of $400 \mathrm{mg} / \mathrm{kg}$ acetaminophen in hamsters could deplete GSH levels, leading to the reduction of enzyme activities of catalase and GPx and exacerbated oxidative stress in the liver.
In addition, $\mathrm{NADPH}$ oxidase (Nox) can act as a pathological link between oxidative stress and endoplasmic reticulum stress, in the process that drives cellular apoptosis [77]. In an experimental rodent model of microsomes incubated with acetaminophen, it was demonstrated that Nox could promote lipid peroxidation, decrease thiol content, including the activity if glutathione S-transferase to facilitate oxidative damage [78]. Interestingly, ablation of Nox4 in mice resulted in impaired homocysteine metabolism and decreased GSH levels, to protect against acetaminophen-induced liver damage in mice [79].

3.1. The Role of Antioxidants in Drug-Induced Liver Injury. Apparently, enhancing intracellular antioxidative systems remains a feasible strategy to counteract oxidative stress to alleviate cellular damage. As such, liver cells respond to oxidative stress by expressing antioxidant and detoxification enzymes to protect against the degenerative effect of free radicals. The expression of these cytoprotective factors is in part regulated by the nuclear factor erythroid 2-related factor 2 (Nrf2), which senses increased ROS production [80] to produce detoxifying antioxidants, a necessary process for retaining redox homeostasis $[81,82]$. Upon activationdue to redox stress, Nrf2 has the capacity to upregulate genes encoding for cytoprotective defence antioxidants such as SOD, GPx, GST, heme oxigenase-1 (HO-1), and NADPH quinone oxidoreductase 1 (NQO1) [81]. Experimental 


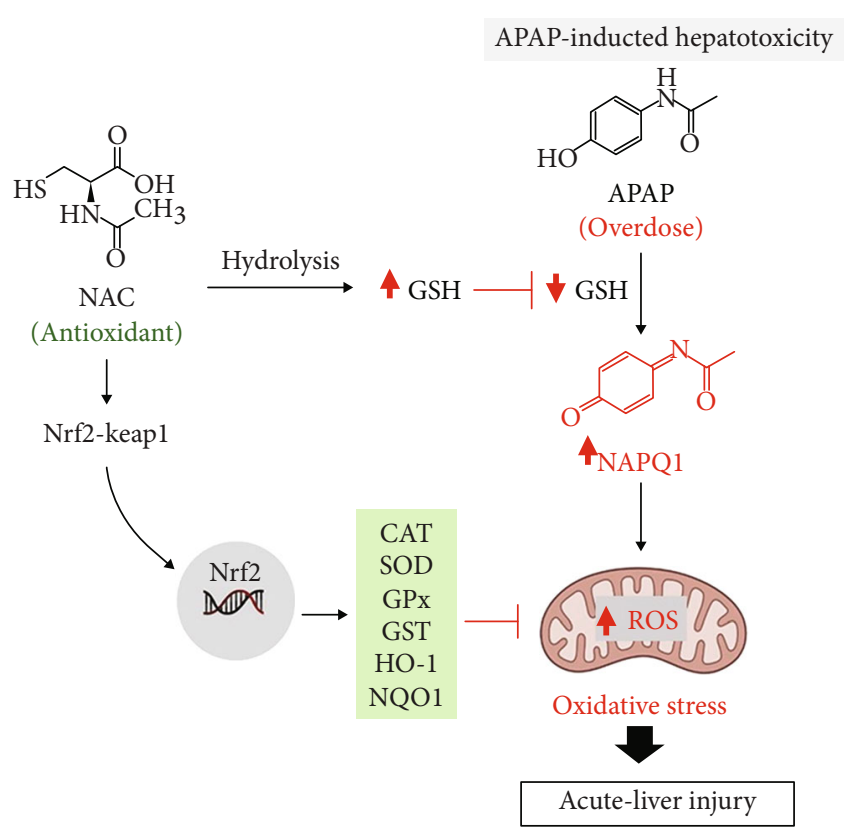

Figure 2: Proposed molecular mechanism by which $\mathrm{N}$-acetyl cysteine (NAC) ameliorates acute liver injury by targeting oxidative stress. Briefly, NAC can enhance the endogenous GSH levels to elicit antioxidant responses, mainly via $\mathrm{Nrf} 2$ activation and its linked antioxidant-detoxifying enzymes like SOD, GPx, GST, HO-1, and NQO1, to protecting against APAP-induced hepatotoxicity. Abbreviations: APAP: paracetamol/acetaminophen; NAPQI: N-acetyl-p-benzo-quinone imine; GSH: reduced glutathione; GSSH: oxidized glutathione; Nrf2: nuclear factor erythroid 2-related factor 2; Keap1: Kelch-like ECH-associated protein 1; CAT: catalase; SOD: superoxide dismutase; GPx: glutathione peroxidase; GST: glutathione s-transferase; HO-1: heme oxigenase-1; NQO1: oxidoreductase 1.

evidence suggest $\mathrm{Nrf} 2$ is essential to protect against liver injury including that may be induced by acetaminophen and other hepatotoxicants [83]. Figure 2 gives an overview of proposed mechanisms by which NAC protects against oxidative stress-induced hepatic injury, in response to paracetamol-toxicity. To further verify the toxic effects of oxidative stress in causing liver injury, Xiao et al. [84] recently demonstrated that generation of advanced oxidation protein products and ischaemia-modified albumin can be used to monitor oxidative stress levels in patients with DILI. Altogether, oxidative stress appears to be at the center of pathophysiological events involved in DILI. Oxidative stress-mediated liver injury includes depletion of hepatic GSH levels, together with the disruption of other antioxidant defence mechanisms, leading to overproduction of ROS, thus provoking hepatocellular damage observed in DILI.

\section{Protective Effects of N-Acetyl Cysteine against Drug-Induced Liver Injury}

As evident from Table 1, a systematic search through major search engines like PubMed and Google Scholar could retrieve 12 relevant RCTs reporting on the impact of NAC infusion on liver function in patients with DILI (Figure 3).
Notably, only studies published between 2003 and 2019 could meet the inclusion criteria, and these were mainly from Australia, Denmark, France, India, United States and United Kingdom. Table 1 gives an overview of RCTs reporting on the effects of NAC on liver function in patients with DILI. The reported evidence covers different aspects of DILI, ranging from paracetamol to nonparacetamol-induced complications, as well as implications of various doses or intervention times with NAC.

From Table 1 evidence, it was clear that most RCTs reported on the therapeutic effects of NAC against paracetamol overdose. This is consistent with overwhelming literature that has been published over the years $[20,85,86]$, supporting the use of NAC to protect against liver injury that is consistent with paracetamol intoxication. For example, in an RCT conducted between 1996 and 1999, Yip and Dart [87] showed that an NAC loading dose of $140 \mathrm{mg} / \mathrm{kg}$ body weight, given in a $20 \mathrm{~h}$ period, was effective in preventing hepatic injury after an acute acetaminophen overdose, especially in patients with an acetaminophen level below the probable hepatotoxicity line. Consistently, Heard et al. [88] showed that NAC loading at $140 \mathrm{mg} / \mathrm{kg}$, followed by $70 \mathrm{mg} / \mathrm{kg}$ every $4 \mathrm{~h}$ for 12 doses, could reduce the rate of hepatotoxicity and adverse events in patients with history of acute acetaminophen ingestion within the $24 \mathrm{~h}$ preceding emergency department evaluation. Alternatively, Pickering et al. [89] showed that an even higher dose of NAC at $300 \mathrm{mg}$ twice daily, given concurrently with paracetamol at $1 \mathrm{~g}$ daily for 4 days, could neutralize paracetamol-induced hepatic toxicity, in part, by maintaining GSH levels.

Others, like Wong et al. [90, 91], went further to assess the impact of dosing differently with NAC on paracetamol overdose. Here, they showed that in subjects receiving $12 \mathrm{~h}$ NAC regimen $(200 \mathrm{mg} / \mathrm{kg}$ over $4 \mathrm{~h}, 50 \mathrm{mg} / \mathrm{kg}$ over $8 \mathrm{~h})$ had similar circulating metabolite concentrations compared to a $20 \mathrm{~h}$ regimen in selected subjects with a low risk of hepatotoxicity. Also, there was no observed liver injury or any effect on levels of ALT or miR-122 expression [90, 91]. Besides assessing the impact of different doses, others reported on how NAC affects paracetamol when used in combination with other therapies. For instance, Morrison et al. [92] showed that NAC infusion at $100 \mathrm{mg} / \mathrm{kg}$ over $2 \mathrm{~h}$, in combination with calmangafodipir, a superoxide dismutase mimetic, could be well tolerated and reduce biomarkers of paracetamol toxicity such as ALT, keratin-18, and circulating caspase-cleaved cytokeratin-18 in patients with paracetamol overdose. Also, in patients with paracetamol poisoning, Bateman et al. [93] aimed to determine the positive effects of administering NAC at $150 \mathrm{mg} / \mathrm{kg}$ for the longer $(20,25 \mathrm{~h})$ or shorter $(12 \mathrm{~h})$ duration, with ondansetron pretreatment $(4 \mathrm{mg})$. Here, patients with paracetamol poisoning, a shorter $(12 \mathrm{~h})$ modified NAC regimen resulted in less vomiting, fewer anaphylactoid reactions, and reduced need for treatment interruption.

Besides paracetamol overdose, other studies reported on other forms of liver injury, such as that induced by excessive alcohol intake. For example, Stewart et al. [94] showed that NAC at $150 \mathrm{mg} / \mathrm{kg}$, followed by $100 \mathrm{mg} / \mathrm{kg} /$ day for 1 week, when combined with vitamins A-E, biotin, selenium, zinc, 


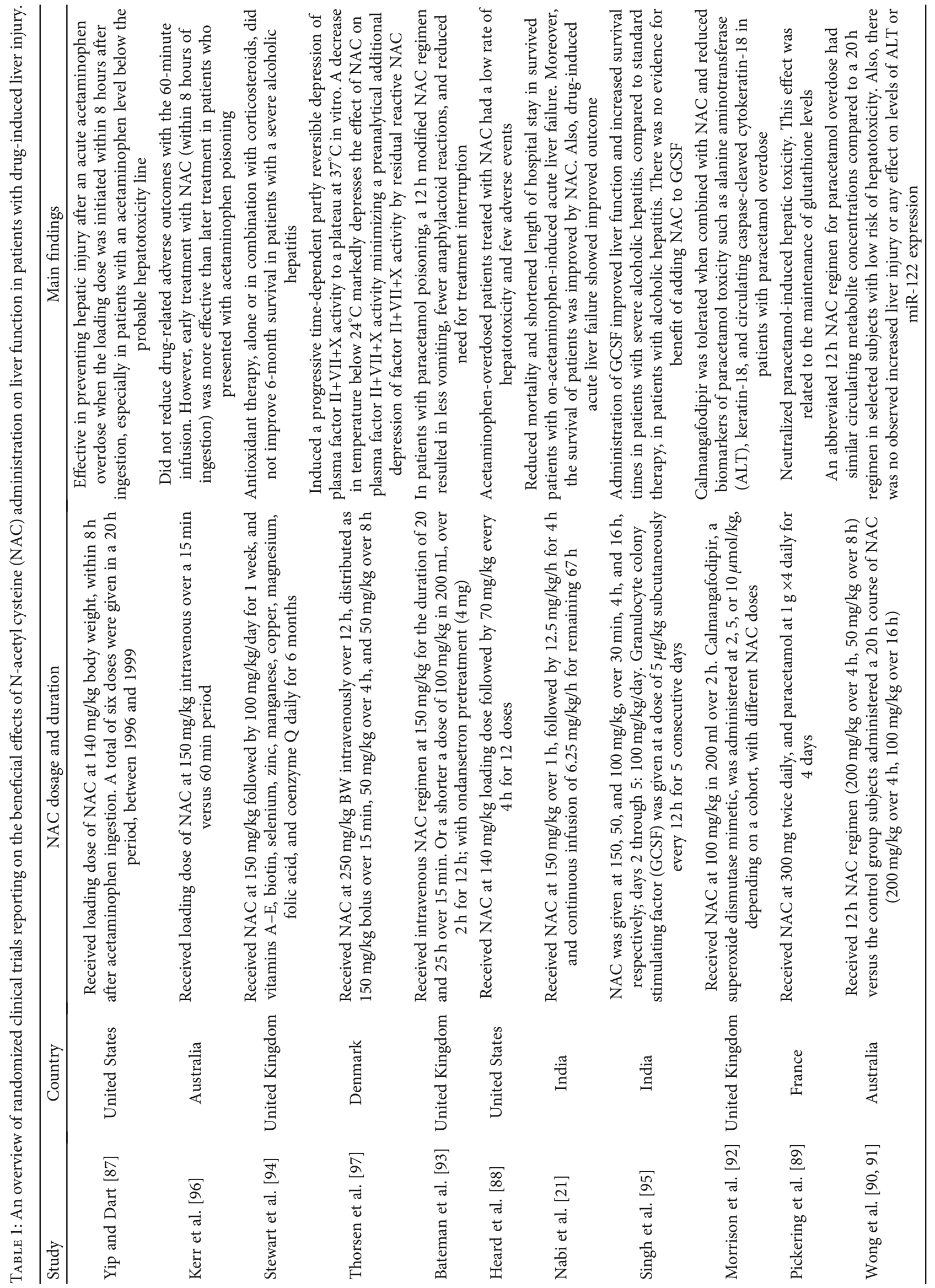




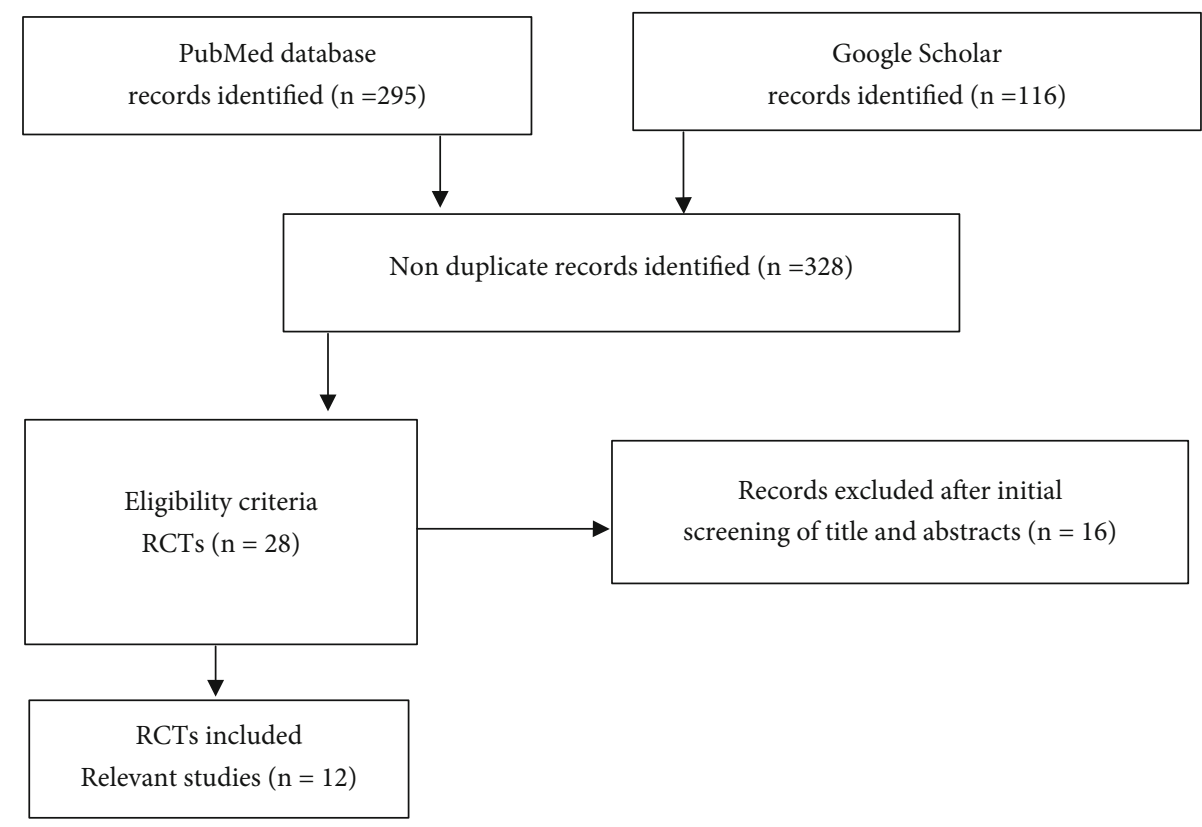

FIGURE 3: The flow diagram, relating to study inclusion criteria. Briefly, a systematic search of literature using major search engines, PubMed and Google Scholar, revealed approximately 12 relevant randomized controlled trials (RCTs) reporting on the impact of N-acetyl cysteine infusion on liver function in patients with drug-induced liver injury.

manganese, copper, magnesium, folic acid, and coenzyme Q daily for 6 months, could not improve survival in patients with a severe alcoholic hepatitis. Singh et al. [95] also demonstrated that there was no benefit of adding NAC, in patients receiving granulocyte colony stimulating factor, as the latter had already displayed enhanced efficacy in improving liver function and increase survival times in patients with severe alcoholic hepatitis. Here, NAC was given at 150, 50, and $100 \mathrm{mg} / \mathrm{kg}$, over $30 \mathrm{~min}, 4 \mathrm{~h}$, and $16 \mathrm{~h}$, respectively; days 2 through 5: $100 \mathrm{mg} / \mathrm{kg} /$ day, whereas granulocyte colony stimulating factor was given at a dose of $5 \mu \mathrm{g} / \mathrm{kg}$ subcutaneously every $12 \mathrm{~h}$ for 5 consecutive days. Perhaps a significant study by Nabi et al. [21] demonstrated that NAC at $150 \mathrm{mg} /$ $\mathrm{kg}$ over $1 \mathrm{~h}$, followed by $12.5 \mathrm{mg} / \mathrm{kg} / \mathrm{h}$ for $4 \mathrm{~h}$ and a continuous infusion of $6.25 \mathrm{mg} / \mathrm{kg} / \mathrm{h}$ for the remaining $67 \mathrm{~h}$, could reduce the mortality and shortened the length of hospital stay in survived patients with nonacetaminophen-induced acute liver failure. This was related to improved survival of patients and drug-induced acute liver failure.

From the evidence summarized in Table 1, it became increasingly relevant to determine how different doses of NAC infusion, including varied treatment duration times, interfere with the efficacy of this antioxidant in blocking drug-induced liver injury. In a study by Kerr et al. [96], they assessed whether the extent of adverse events caused by intravenous NAC at $150 \mathrm{mg} / \mathrm{kg}$, when the initial dose is received over a $60 \mathrm{~min}$ period compared with the standard infusion period of $15 \mathrm{~min}$. The results showed that early treatment with NAC was more effective than later treatment in patients who presented with acetaminophen poisoning [96]. Thorsen et al. [97] showed that an average NAC dose of $250 \mathrm{mg} / \mathrm{kg}$ body weight over $12 \mathrm{~h}$, distributed as $150 \mathrm{mg} /$ $\mathrm{kg}$ bolus over $15 \mathrm{~min}, 50 \mathrm{mg} / \mathrm{kg}$ over $4 \mathrm{~h}$, and $50 \mathrm{mg} / \mathrm{kg}$ over
$8 \mathrm{~h}$, could induce a progressive time-dependent partly reversible depression of plasma factor II+VII+X activity, which is a significant prognostic marker for the severity of liver damage in paracetamol-poisoned patients. Thus, suggesting that beyond enhancing hepatic GSH levels, other therapeutic mechanisms may be exerted by NAC to alleviate drug-induced liver injury. Worthy to note is that, in addition to its protective potential against hepatotoxicity, NAC also has a pharmacological role as a mucolytic agent due to its ability to break the disulphide bridges of the highmolecular-weight glycoproteins in the mucus which in turn results in reduced viscosity [98]. In fact, growing preclinical evidence indicates NAC may plan an important role in ameliorating complications linked with obesity or cardiovascular disease, and this is especially due to its capacity to ameliorate the detrimental effects of oxidative stress and inflammation $[17,22,23]$.

\section{Summary and Future Perspectives}

Currently, it is understood that depletion of intracellular antioxidant defences, especially the levels of GSH remains the critical factor implicated in the worsening of hepatic injury, in response to drug overdose. GSH is regarded as one of the major cytoprotective antioxidants through its direct and indirect scavenging effects on ROS, leading to the amelioration of oxidative stress and improved liver function $[99,100]$. Furthermore, current research indicates that exogenous administration of GSH in mice can also protect against acetaminophen-induced liver injury [101]. Perhaps, also highlighting the therapeutic significance of NAC in protecting against DILI, as an established precursor for endogenous GSH synthesis [71]. Clinical evidence summarized in 
the current review actually validates decades of literature [102] on beneficial effects of NAC in reducing the mortality of patients with DILI. Table 1 indicates that, besides acetaminophen, NAC can also improve liver function in relation to other forms of DILI such as those induced by excessive alcohol intake [21]. The implicated therapeutic mechanisms of NAC extend from enhancing hepatic GSH levels to reducing biomarkers of paracetamol toxicity such as keratin-18 and circulating caspase-cleaved cytokeratin-18 [92]. However, although such benefits with NAC infusions are observed in patients with DILI, other draw backs have also been reported. Clearly, additional RCTs are still required to confirm whether NAC administration can provide synergistic effect when combined with other therapies to improve liver function in patients DILI. Equally, additional evidence is still necessary to determine whether changing commonly doses and treatment duration times can be more beneficial than using a standard treatment regimen, relevant to protecting against DILI.

\section{Abbreviations}

$\begin{array}{ll}\text { ALP: } & \text { Alkaline phosphatase } \\ \text { ALT: } & \text { Alanine aminotransferase } \\ \text { AST: } & \text { Aspartate aminotransferase } \\ \text { CAT: } & \text { Catalase } \\ \text { CYP: } & \text { Cytochrome p450 } \\ \text { DILI: } & \text { Drug-induced liver injury } \\ \text { ETC: } & \text { Electron transport chain } \\ \text { ER: } & \text { Endoplasmic reticulum } \\ \text { GPx: } & \text { Glutathione peroxidase } \\ \text { GSH: } & \text { Glutathione } \\ \text { HO-1: } & \text { Heme oxigenase-1 } \\ \text { JNK: } & \text { c-Jun N-terminal kinase } \\ \text { Keap1: } & \text { Kelch-like ECH-associated protein 1 } \\ \text { NAC: } & \text { N-Acetyl cysteine } \\ \text { NAPQI: } & \text { N-Acetyl-p-benzo-quinone imine } \\ \text { Nox: } & \text { NADPH oxidase } \\ \text { NQO1: } & \text { Oxidoreductase 1 } \\ \text { RCTs: } & \text { Randomized controlled trials } \\ \text { RCT: } & \text { Randomized controlled trials } \\ \text { ROS: } & \text { Reactive oxygen species } \\ \text { SOD: } & \text { Superoxide dismutase. } \\ & \end{array}$

\section{Data Availability}

Data related to search strategy, study selection, and extraction items will be made available upon request after the manuscript is published.

\section{Consent}

No individual person's data has been included in this manuscript.

\section{Disclosure}

The content hereof is the sole responsibility of the authors and do not necessarily represent the official views of the funders, NRF, or the SAMRC.

\section{Conflicts of Interest}

The authors declare no conflict of interest.

\section{Authors' Contributions}

$\mathrm{YN}, \mathrm{KZ}$, and PVD wrote the concept and original draft. YN, $\mathrm{KZ}$, and PVD worked on literature search and data extraction. YN, KZ, NC, BBN, TMN, SEM-M, KBG, FM, LT, and PVD are responsible for the writing and final approval of the manuscript.

\section{Acknowledgments}

This work was supported in part by baseline funding from the Biomedical Research and Innovation Platform of the South African Medical Research Council (SAMRC) and the National Research Foundation (Grant numbers: 128107 and 117829). Y Ntamo is a postdoctoral research fellow, funded by the Department of Science and Technology (DST) and the National Research Foundation (NRF): Professional Development Programme Block Grant (Grant number: 128107). The work by K Ziqubu, reported herein, was made possible through partial funding by the South African Medical Research Council through its Division of Research Capacity Development under the Researcher Development Award Programme. BB Nkambule is a University of KwaZulu-Natal Developing Research Innovation, Localisation and Leadership in South Africa (DRILL) fellow. DRILL is a NIH D43 grant (D43TW010131) awarded to UKZN in 2015 to support a research training and induction programme for early career academics.

\section{References}

[1] S. David and J. P. Hamilton, "Drug-induced liver injury," US gastroenterology \& hepatology review, vol. 6, pp. 73-80, 2010.

[2] R. J. Andrade, A. Ortega-Alonso, and M. I. Lucena, “Druginduced liver injury clinical consortia: a global research response for a worldwide health challenge," Expert Opinion on Drug Metabolism \& Toxicology, vol. 12, no. 6, pp. 589593, 2016.

[3] A. J. Macpherson, M. Heikenwalder, and S. C. Ganal-Vonarburg, "The liver at the nexus of host-microbial interactions," Cell Host \& Microbe, vol. 20, no. 5, pp. 561-571, 2016.

[4] E. G. Giannini, R. Testa, and V. Savarino, "Liver enzyme alteration: a guide for clinicians," CMAJ, vol. 172, no. 3, pp. 367-379, 2005.

[5] R. J. Andrade, M. Robles, A. Fernández-Castañer, S. LópezOrtega, M. C. López-Vega, and M. I. Lucena, "Assessment of drug-induced hepatotoxicity in clinical practice: a challenge for gastroenterologists," World Journal of Gastroenterology, vol. 13, no. 3, pp. 329-340, 2007.

[6] Council for International Organizations of Medical Sciences, Drug-induced liver injury (DILI): Current status and future directions for drug development and the post-market setting, World Health Organization, 2020.

[7] M. D. Leise, J. J. Poterucha, and J. A. Talwalkar, "Druginduced liver injury," Mayo Clinic Proceedings, vol. 89, no. 1, pp. 95-106, 2014. 
[8] R. J. Andrade, N. Chalasani, E. S. Björnsson et al., "Druginduced liver injury," Nature Reviews. Disease Primers, vol. 5, no. 1, p. 58, 2019.

[9] S. Fu, D. Wu, W. Jiang et al., "Molecular biomarkers in druginduced liver injury: challenges and future perspectives," Frontiers in Pharmacology, vol. 10, p. 1667, 2019.

[10] M. Robles-Díaz, I. Medina-Caliz, C. Stephens, R. J. Andrade, and M. I. Lucena, "Biomarkers in DILI: One More Step Forward," Frontiers in Pharmacology, vol. 7, p. 267, 2016.

[11] K. Reyes-Gordillo, R. Shah, and P. Muriel, "Oxidative stress and inflammation in hepatic diseases: current and future therapy," Oxidative Medicine and Cellular Longevity, vol. 2017, 2017.

[12] E. Albano, M. Rundgren, P. J. Harvison, S. D. Nelson, and P. Moldéus, "Mechanisms of N-acetyl-p-benzoquinone imine cytotoxicity," Molecular Pharmacology, vol. 28, no. 3, pp. 306-311, 1985.

[13] S. Casas-Grajales and P. Muriel, "Antioxidants in liver health," World Journal of Gastrointestinal Pharmacology and Therapeutics, vol. 6, no. 3, pp. 59-72, 2015.

[14] R. Pillon Barcelos, L. F. Freire Royes, J. Gonzalez-Gallego, and G. Bresciani, "Oxidative stress and inflammation: liver responses and adaptations to acute and regular exercise," Free Radical Research, vol. 51, no. 2, pp. 222-236, 2017.

[15] E. Kheradpezhouh, L. Ma, A. Morphett, G. J. Barritt, and G. Y. Rychkov, "TRPM2 channels mediate acetaminopheninduced liver damage," Proceedings of the National Academy of Sciences of the United States of America, vol. 111, no. 8, pp. 3176-3181, 2014.

[16] I. Cirilli, P. Orlando, F. Marcheggiani et al., "The protective role of bioactive Quinones in stress-induced senescence phenotype of endothelial cells exposed to cigarette smoke extract," Antioxidants, vol. 9, no. 10, p. 1008, 2020.

[17] P. V. Dludla, S. E. Mazibuko-Mbeje, T. M. Nyambuya et al., "The beneficial effects of N-acetyl cysteine (NAC) against obesity associated complications: a systematic review of preclinical studies," Pharmacological Research, vol. 146, article 104332, 2019.

[18] E. Asevedo, A. C. Mendes, M. Berk, and E. Brietzke, "Systematic review of $\mathrm{N}$-acetylcysteine in the treatment of addictions," Revista Brasileira de Psiquiatria, vol. 36, no. 2, pp. 168-175, 2014.

[19] Š. Šalamon, B. Kramar, T. P. Marolt, B. Poljšak, and I. Milisav, "Medical and dietary uses of N-acetylcysteine," Antioxidants, vol. 8, no. 5, p. 111, 2019.

[20] G. G. Liou, C. C. Hsieh, Y. J. Lee et al., "N-acetyl cysteine overdose inducing hepatic steatosis and systemic inflammation in both Propacetamol-induced hepatotoxic and Normal mice," Antioxidants, vol. 10, no. 3, p. 442, 2021.

[21] T. Nabi, S. Nabi, N. Rafiq, and A. Shah, "Role of Nacetylcysteine treatment in non-acetaminophen-induced acute liver failure: a prospective study," Saudi Journal of Gastroenterology, vol. 23, no. 3, pp. 169-175, 2017.

[22] P. V. Dludla, B. B. Nkambule, S. C. Dias, and R. Johnson, "Cardioprotective potential of $\mathrm{N}$-acetyl cysteine against hyperglycaemia-induced oxidative damage: a protocol for a systematic review," Systematic Reviews, vol. 6, no. 1, p. 96, 2017.

[23] P. V. Dludla, S. C. Dias, N. Obonye, R. Johnson, J. Louw, and B. B. Nkambule, "A systematic review on the protective effect of $\mathrm{N}$-acetyl cysteine against diabetes-associated cardiovascu- lar complications," American Journal of Cardiovascular Drugs, vol. 18, no. 4, pp. 283-298, 2018.

[24] M. Chen, H. Bisgin, L. Tong et al., "Toward predictive models for drug-induced liver injury in humans: are we there yet?" Biomarkers in Medicine, vol. 8, no. 2, pp. 201-213, 2014.

[25] I. Khanna, "Drug discovery in pharmaceutical industry: productivity challenges and trends," Drug Discovery Today, vol. 17, no. 19-20, pp. 1088-1102, 2012.

[26] G. Abboud and N. Kaplowitz, "Drug-induced liver injury," Drug Safety, vol. 30, no. 4, pp. 277-294, 2007.

[27] L. P. James, P. R. Mayeux, and J. A. Hinson, "Acetaminophen-induced hepatotoxicity," Drug Metabolism and Disposition, vol. 31, no. 12, pp. 1499-1506, 2003.

[28] N. Kaplowitz, "Drug-induced liver injury," Clinical Infectious Diseases, vol. 38, Supplement 2, pp. S44-S48, 2004.

[29] L. J. Chun, M. J. Tong, R. W. Busuttil, and J. R. Hiatt, “Acetaminophen hepatotoxicity and acute liver failure," Journal of Clinical Gastroenterology, vol. 43, no. 4, pp. 342-349, 2009.

[30] H. Devarbhavi, "An update on drug-induced liver injury," Journal of Clinical and Experimental Hepatology, vol. 2, no. 3, pp. 247-259, 2012.

[31] T. Shen, Y. Liu, J. Shang et al., "Incidence and etiology of drug-induced liver injury in mainland China," Gastroenterology, vol. 156, no. 8, pp. 2230-2241.e11, 2019.

[32] E. S. Björnsson, O. M. Bergmann, H. K. Björnsson, R. B. Kvaran, and S. Olafsson, "Incidence, presentation, and outcomes in patients with drug-induced liver injury in the general population of Iceland," Gastroenterology, vol. 144, no. 7, pp. 1419-1425.e3, 2013.

[33] R. J. Fontana, L. B. Seeff, R. J. Andrade et al., "Standardization of nomenclature and causality assessment in drug-induced liver injury: summary of a clinical research workshop," Hepatology, vol. 52, no. 2, pp. 730-742, 2010.

[34] H. Ye, L. J. Nelson, M. G. . Moral, E. Martínez-Naves, and F. J. Cubero, "Dissecting the molecular pathophysiology of druginduced liver injury," World Journal of Gastroenterology, vol. 24, no. 13, pp. 1373-1385, 2018.

[35] L. Boone, D. Meyer, P. Cusick et al., "Selection and interpretation of clinical pathology indicators of hepatic injury in preclinical studies," Veterinary Clinical Pathology, vol. 34, no. 3, pp. 182-188, 2005.

[36] R. A. Mitchell, S. Rathi, M. Dahiya, J. Zhu, T. Hussaini, and E. M. Yoshida, "Public awareness of acetaminophen and risks of drug induced liver injury: results of a large outpatient clinic survey," PLoS One, vol. 15, no. 3, article e0229070, 2020.

[37] H. Jaeschke, J. Y. Akakpo, D. S. Umbaugh, and A. Ramachandran, "Novel therapeutic approaches against acetaminophen-induced liver injury and acute liver failure," Toxicological Sciences, vol. 174, no. 2, pp. 159-167, 2020.

[38] M. E. Zoubek, M. I. Lucena, R. J. Andrade, and C. Stephens, "Systematic review: ibuprofen-induced liver injury," Alimentary Pharmacology \& Therapeutics, vol. 51, no. 6, pp. 603$611,2020$.

[39] R. Kumar, Shalimar, V. Bhatia et al., "Antituberculosis therapy-induced acute liver failure: magnitude, profile, prognosis, and predictors of outcome," Hepatology, vol. 51, no. 5, pp. 1665-1674, 2010.

[40] H. Devarbhavi, R. Dierkhising, W. K. Kremers, M. S. Sandeep, D. Karanth, and C. K. Adarsh, "Single-center experience with drug-induced liver injury from India: causes, outcome, prognosis, and predictors of mortality," The 
American Journal of Gastroenterology, vol. 105, no. 11, pp. 2396-2404, 2010.

[41] B. Vincenzi, G. Armento, M. Spalato Ceruso et al., "Druginduced hepatotoxicity in cancer patients - implication for treatment," Expert Opinion on Drug Safety, vol. 15, no. 9, pp. 1219-1238, 2016.

[42] A. Pieniążek, J. Czepas, J. Piasecka-Zelga, K. Gwoździński, and A. Koceva-Chyła, "Oxidative stress induced in rat liver by anticancer drugs doxorubicin, paclitaxel and docetaxel," Advances in Medical Sciences, vol. 58, no. 1, pp. 104-111, 2013.

[43] D. Remash, D. S. Prince, C. McKenzie, S. I. Strasser, S. Kao, and K. Liu, "Immune checkpoint inhibitor-related hepatotoxicity: a review," World Journal of Gastroenterology, vol. 27, no. 32, pp. 5376-5391, 2021.

[44] O. Oyebode, N. B. Kandala, P. J. Chilton, and R. J. Lilford, "Use of traditional medicine in middle-income countries: a WHO-SAGE study," Health Policy and Planning, vol. 31, no. 8, pp. 984-991, 2016.

[45] R. Teschke and A. Eickhoff, "Herbal hepatotoxicity in traditional and modern medicine: actual key issues and new encouraging steps," Frontiers in Pharmacology, vol. 6, p. 72, 2015.

[46] A. K. Upadhyay, K. Kumar, A. Kumar, and H. S. Mishra, "Tinospora cordifolia (Willd.) Hook. f. and Thoms. (Guduchi) - Validation of the Ayurvedic pharmacology through experimental and clinical studies," International Journal of Ayurveda Research, vol. 1, no. 2, pp. 112-121, 2010.

[47] M. Porceddu, N. Buron, C. Roussel, G. Labbe, B. Fromenty, and A. Borgne-Sanchez, "Prediction of liver injury induced by chemicals in human with a multiparametric assay on isolated mouse liver mitochondria," Toxicological Sciences, vol. 129, no. 2, pp. 332-345, 2012.

[48] A. Abid, F. Subhani, F. Kayani, S. Awan, and S. Abid, "Drug induced liver injury is associated with high mortality-a study from a tertiary care hospital in Pakistan," PLoS One, vol. 15, no. 4, article e0231398, 2020.

[49] C. Sgro, F. Clinard, K. Ouazir et al., "Incidence of druginduced hepatic injuries: a French population-based study," Hepatology, vol. 36, no. 2, pp. 451-455, 2002.

[50] H. Dancygier, "Hepatic Biotransformation," in Clinical Hepatology, Springer, Berlin, Heidelberg, 2010.

[51] L. Yu, "Pathogenesis of Liver Injury and Hepatic Failure," in Artificial Liver, L. Li, Ed., Springer, Singapore, 2021.

[52] M. R. McGill and H. Jaeschke, "Animal models of druginduced liver injury," Biochimica et Biophysica Acta - Molecular Basis of Disease, vol. 1865, no. 5, pp. 1031-1039, 2019.

[53] H. Jaeschke, Y. Xie, and M. R. McGill, "Acetaminopheninduced liver injury: from animal models to humans," Journal of Clinical and Translational Hepatology, vol. 2, no. 3, pp. 153-161, 2014.

[54] Y. Pan, M. Cao, D. You, G. Qin, and Z. Liu, "Research Progress on the animal models of drug-induced liver injury: current status and further perspectives," BioMed Research International, vol. 2019, Article ID 1283824, 2019.

[55] V. Vilas-Boas, A. Cooreman, E. Gijbels et al., "Primary hepatocytes and their cultures for the testing of druginduced liver injury," Advances in Pharmacology, vol. 85, pp. 1-30, 2019.

[56] G. Elaut, T. Henkens, P. Papeleu et al., "Molecular mechanisms underlying the dedifferentiation process of isolated hepatocytes and their cultures," Current Drug Metabolism, vol. 7, no. 6, pp. 629-660, 2006.

[57] D. T. Scadden, "The stem-cell niche as an entity of action," Nature, vol. 441, no. 7097, pp. 1075-1079, 2006.

[58] R. Chang, J. Nam, and W. Sun, "Direct cell writing of 3D microorgan for in vitro pharmacokinetic model," Tissue Engineering. Part C, Methods, vol. 14, no. 2, pp. 157-166, 2008.

[59] J. W. Haycock, "3D cell culture: a review of current approaches and techniques," Methods in Molecular Biology, vol. 695, pp. 1-15, 2011.

[60] M. Ravi, V. Paramesh, S. R. Kaviya, E. Anuradha, and F. D. P. Solomon, "3D cell culture systems: advantages and applications," Journal of Cellular Physiology, vol. 230, no. 1, pp. 16-26, 2015.

[61] N. Jacobi, R. Seeboeck, E. Hofmann et al., "Organotypic three-dimensional cancer cell cultures mirror drug responsesin vivo: lessons learned from the inhibition of EGFR signaling," Oncotarget, vol. 8, no. 64, pp. $107423-$ 107440, 2017.

[62] Y. Ntamo, E. Samodien, J. Burger, N. Muller, C. J. Muller, and N. Chellan, "In vitro characterization of insulin-producing $\beta$ cell spheroids," Frontiers in Cell and Development Biology, vol. 8, article 623889, 2020.

[63] J. Jiang, J. E. J. Wolters, S. G. van Breda, J. C. Kleinjans, and T. M. de Kok, "Development of novel tools for the in vitro investigation of drug-induced liver injury," Expert Opinion on Drug Metabolism \& Toxicology, vol. 11, no. 10, pp. 1523-1537, 2015.

[64] H. S. Schadt, A. Wolf, F. Pognan, S. D. Chibout, M. Merz, and G. A. Kullak-Ublick, "Bile acids in drug induced liver injury: key players and surrogate markers," Clinics and Research in Hepatology and Gastroenterology, vol. 40, no. 3, pp. 257266, 2016.

[65] P. V. Dludla, B. B. Nkambule, S. E. Mazibuko-Mbeje et al., "N-acetyl cysteine targets hepatic lipid accumulation to curb oxidative stress and inflammation in NAFLD: a comprehensive analysis of the literature," Antioxidants, vol. 9, no. 12, p. 1283, 2020.

[66] M. Villanueva-Paz, L. Morán, N. López-Alcántara et al., "Oxidative stress in drug-induced liver injury (DILI): from mechanisms to biomarkers for use in clinical practice," Antioxidants, vol. 10, no. 3, p. 390, 2021.

[67] M. Arconzo, E. Piccinin, and A. Moschetta, "Increased risk of acute liver failure by pain killer drugs in NAFLD: focus on nuclear receptors and their coactivators," Digestive and Liver Disease, vol. 53, no. 1, pp. 26-34, 2021.

[68] D. Pessayre, B. Fromenty, A. Berson et al., "Central role of mitochondria in drug-induced liver injury," Drug Metabolism Reviews, vol. 44, no. 1, pp. 34-87, 2012.

[69] A. Weidinger and A. V. Kozlov, "Biological activities of reactive oxygen and nitrogen species: oxidative stress versus signal transduction," Biomolecules, vol. 5, no. 2, pp. 472-484, 2015.

[70] A. M. Larson, J. Polson, R. J. Fontana et al., “Acetaminopheninduced acute liver failure: results of a United States multicenter, prospective study," Hepatology, vol. 42, no. 6, pp. 1364-1372, 2005.

[71] T. B. Jeong, J. H. Kim, S. H. Kim et al., "Comparison of toxic responses to acetaminophen challenge in ICR mice originating from different sources," Lab Anim Res, vol. 35, no. 1, p. $16,2019$. 
[72] A. Berson, S. Renault, P. Letteron et al., "Uncoupling of rat and human mitochondria: A possible explanation for tacrineinduced liver dysfunction," Gastroenterology, vol. 110, no. 6, pp. 1878-1890, 1996.

[73] H. Jaeschke, T. R. Knight, and M. L. Bajt, "The role of oxidant stress and reactive nitrogen species in acetaminophen hepatotoxicity," Toxicology Letters, vol. 144, no. 3, pp. 279-288, 2003.

[74] K. Wang, "Molecular mechanisms of hepatic apoptosis regulated by nuclear factors," Cellular Signalling, vol. 27, no. 4, pp. 729-738, 2015.

[75] L. Longato, K. Ripp, M. Setshedi et al., "Insulin resistance, ceramide accumulation, and endoplasmic reticulum stress in human chronic alcohol-related liver disease," Oxidative Medicine and Cellular Longevity, vol. 2012, Article ID 479348, 2012.

[76] E. S. Jin, M. H. Lee, and C. R. Malloy, "Divergent effects of glutathione depletion on isocitrate dehydrogenase 1 and the pentose phosphate pathway in hamster liver," Physiological Reports, vol. 8, no. 16, article e14554, 2020.

[77] G. Li, C. Scull, L. Ozcan, and I. Tabas, "NADPH oxidase links endoplasmic reticulum stress, oxidative stress, and PKR activation to induce apoptosis," The Journal of Cell Biology, vol. 191, no. 6, pp. 1113-1125, 2010.

[78] M. E. Letelier, M. López-Valladares, L. Peredo-Silva, D. Rojas-Sepúlveda, and P. Aracena, "Microsomal oxidative damage promoted by acetaminophen metabolism," Toxicology In Vitro, vol. 25, no. 7, pp. 1310-1313, 2011.

[79] T. V. Murray, X. Dong, G. J. Sawyer et al., "NADPH oxidase 4 regulates homocysteine metabolism and protects against acetaminophen-induced liver damage in mice," Free Radical Biology \& Medicine, vol. 89, pp. 918-930, 2015.

[80] S. Kasai, S. Shimizu, Y. Tatara, J. Mimura, and K. Itoh, "Regulation of Nrf2 by mitochondrial reactive oxygen species in physiology and pathology," Biomolecules, vol. 10, no. 2, p. 320, 2020.

[81] Q. Ma, "Role of nrf2 in oxidative stress and toxicity," Annual Review of Pharmacology and Toxicology, vol. 53, pp. 401-426, 2013.

[82] P. V. Dludla, C. Muller, E. Joubert et al., “Aspalathin protects the heart against hyperglycemia-induced oxidative damage by up-regulating Nrf2 expression," Molecules, vol. 22, no. 1, p. 129, 2017.

[83] J. Liu, K. C. Wu, Y. F. Lu, E. Ekuase, and C. D. Klaassen, "Nrf2 protection against liver injury produced by various hepatotoxicants," Oxidative Medicine and Cellular Longevity, vol. 2013, Article ID 305861, 2013.

[84] L. L. Xiao, F. Zhang, Y. L. Zhao et al., "Using advanced oxidation protein products and ischaemia-modified albumin to monitor oxidative stress levels in patients with druginduced liver injury," Scientific Reports, vol. 10, no. 1, p. $18128,2020$.

[85] W. S. Waring, "Novel acetylcysteine regimens for treatment of paracetamol overdose," Therapeutic advances in drug safety, vol. 3, no. 6, pp. 305-315, 2012.

[86] K. J. Heard, "Acetylcysteine for acetaminophen poisoning," The New England Journal of Medicine, vol. 359, no. 3, pp. 285-292, 2008.

[87] L. Yip and R. C. Dart, "A 20-hour treatment for acute acetaminophen overdose," The New England Journal of Medicine, vol. 348 , no. 24 , pp. 2471-2472, 2003.
[88] K. Heard, B. H. Rumack, J. L. Green et al., “A single-arm clinical trial of a 48-hour intravenous $\mathrm{N}$-acetylcysteine protocol for treatment of acetaminophen poisoning," Clinical Toxicology, vol. 52, no. 5, pp. 512-518, 2014.

[89] G. Pickering, N. Macian, I. Papet, C. Dualé, C. Coudert, and B. Pereira, "N-acetylcysteine prevents glutathione decrease and does not interfere with paracetamol antinociceptive effect at therapeutic dosage: a randomized double-blind controlled trial in healthy subjects," Fundamental \& Clinical Pharmacology, vol. 33, no. 3, pp. 303-311, 2019.

[90] A. Wong, N. Homer, J. W. Dear, K. W. Choy, J. Doery, and A. Graudins, "Paracetamol metabolite concentrations following low risk overdose treated with an abbreviated 12 -h versus 20-h acetylcysteine infusion," Clinical Toxicology (Philadelphia, Pa.), vol. 57, no. 5, pp. 312-317, 2019.

[91] A. Wong, C. Nejad, M. Gantier, K. W. Choy, J. Doery, and A. Graudins, "MicroRNA from a 12 -h versus 20 -h acetylcysteine infusion for paracetamol overdose," Human \& Experimental Toxicology, vol. 38, no. 6, pp. 646-654, 2019.

[92] E. E. Morrison, K. Oatey, B. Gallagher et al., "Principal results of a randomised open label exploratory, safety and tolerability study with calmangafodipir in patients treated with a 12 $\mathrm{h}$ regimen of $\mathrm{N}$-acetylcysteine for paracetamol overdose (POP trial)," eBioMedicine, vol. 46, pp. 423-430, 2019.

[93] D. N. Bateman, J. W. Dear, H. K. R. Thanacoody et al., "Reduction of adverse effects from intravenous acetylcysteine treatment for paracetamol poisoning: a randomised controlled trial," The Lancet, vol. 383, no. 9918, pp. 697-704, 2014.

[94] S. Stewart, M. Prince, M. Bassendine et al., "A randomized trial of antioxidant therapy alone or with corticosteroids in acute alcoholic hepatitis," Journal of Hepatology, vol. 47, no. 2, pp. 277-283, 2007.

[95] V. Singh, A. Keisham, A. Bhalla et al., "Efficacy of Granulocyte Colony-Stimulating Factor and _ $\mathrm{N}_{-}$-Acetylcysteine Therapies in Patients With Severe Alcoholic Hepatitis," Clinical Gastroenterology and Hepatology, vol. 16, no. 10, pp. 1650-1656.e2, 2018.

[96] F. Kerr, A. Dawson, I. M. Whyte et al., “The Australasian Clinical Toxicology Investigators Collaboration Randomized Trial of Different Loading Infusion Rates of_N_-Acetylcysteine," Annals of Emergency Medicine, vol. 45, no. 4, pp. 402 408, 2005.

[97] S. Thorsen, A. Teisner, S. A. Jensen, M. Philips, K. Dalhoff, and F. Bendtsen, "Effect of N-acetylcysteine on the accuracy of the prothrombin time assay of plasma coagulation factor $\mathrm{II}+\mathrm{VII}+\mathrm{X}$ activity in subjects infused with the drug. Influence of time and temperature," Scandinavian Journal of Clinical and Laboratory Investigation, vol. 69, no. 6, pp. 643-650, 2009.

[98] G. Aldini, A. Altomare, G. Baron et al., "N-acetylcysteine as an antioxidant and disulphide breaking agent: the reasons why," Free Radical Research, vol. 52, no. 7, pp. 751762, 2018.

[99] K. Aquilano, S. Baldelli, and M. R. Ciriolo, "Glutathione: new roles in redox signaling for an old antioxidant," Frontiers in Pharmacology, vol. 5, p. 196, 2014.

[100] D. H. Kwon, H. J. Cha, H. Lee et al., "Protective effect of glutathione against oxidative stress-induced cytotoxicity in RAW 264.7 macrophages through activating the nuclear factor erythroid 2-related Factor-2/Heme Oxygenase-1 pathway," Antioxidants, vol. 8, no. 4, p. 82, 2019. 
[101] Y. Masubuchi, J. Nakayama, and Y. Sadakata, "Protective effects of exogenous glutathione and related thiol compounds against drug-induced liver injury," Biological \& Pharmaceutical Bulletin, vol. 34, no. 3, pp. 366-370, 2011.

[102] M. F. Chughlay, N. Kramer, C. W. Spearman, M. Werfalli, and K. Cohen, "N-acetylcysteine for non-paracetamol drug-induced liver injury: a systematic review," British Journal of Clinical Pharmacology, vol. 81, no. 6, pp. 1021-1029, 2016. 